\title{
AN ENERGY BASED SECURITY MEASURE FOR ASSESSING VULNERABILITY TO VOLTAGE COLLAPSE
}

\author{
Christopher L. DeMarco \\ Member, IEEE \\ Thomas J. Overbye \\ Student Member, IEEE \\ Department of Electrical and Computer Engineering \\ University of Wisconsin-Madison \\ Madison, WI 53706
}

\section{ABSTRACT}

This paper defines a security measure to indicate vulnerability to voltage collapse based on an energy function for system models that include voltage variation and reactive loads. The system dynamic model, the energy function and the security measure are first motivated in a simple radial system. Application of the new measure and its computational aspects are then examined in a standard 30 bus example (New England System). The new measure captures nonlinear effects such as var limits on generators that can influence the systems vulnerability to collapse. The behavior of the index with respect to network load increases is nearly linear over a wide range of load variation, facilitating prediction of the onset of collapse.

KEYWORDS: Voltage collapse, voltage stability, energy functions, Lyapunov functions, security assessment.

\section{BACKGROUND AND MOTIVATION}

A large portion of the recent literature devoted to voltage collapse has had as its goal identifying the threshold of voltage collapse, or more generally, developing a security measure to quantify how "close" a particular operating point is to voltage collapse. The crucial point in judging the effectiveness of the later methods is whether or not the "distance" of a given operating point to voltage collapse is physically reasonable and can provide planners and operators with an indication of when corrective control action is necessary. The goal of the work to be presented here is to introduce an energy based measure of proximity to collapse. The method will be motivated by examining a single line example in detail, first in a static setting, and then extended to the desired dynamic framework. This will be followed by application of the energy function approach to the New England 30 bus test system that has been used in the literature to evaluate other voltage collapse indices. Application to this 30 bus system includes a discussion of the computational issues and the uses of the method.

89 SM 712-1 PWRS A paper recoamended and approved by the IEEE Power System Engineering Committee of the IEEE Power Engineering Society for presentation at the IEEE/PES 1989 Summer Meeting, Long Beach, California, July $9-14,1989$. Manuscript subnitted May 18, 1989; made available for printing June 7, 1989.

\section{STATIC FRAMEWORK FOR ONE LINE SYSTEM}

To motivate the energy based method, we begin by examining the static powerflow in a single line example. Consider a system with a single series transmission line (ignore shunts) connecting two buses, numbered 1 and 2 . Bus 1 is treated as a slack bus, with voltage magnitude fixed at 1.0 pu. For simplicity, we will assume that the transmission line is lossless, so that real power injection at bus 1 must equal real power consumed at bus 2 . Also, we will assume that a load is attached at bus 2 , and is represented as constant $\mathrm{P}-\mathrm{Q}$ demand. The following analysis extends easily to the case of $P$ and $Q$ specified as functions of bus voltage. The resulting power balance equations at bus 2 are:

$$
\begin{aligned}
& P_{L}-B_{12} V \sin (\alpha)=0 \\
& Q_{L}-B_{22} V^{2}-B_{12} V \cos (\alpha)=0
\end{aligned}
$$

where

$$
\begin{aligned}
& V:=\text { bus voltage magnitude at bus } 2 \\
& \alpha:=\delta_{1}-\delta_{2}=\text { phase angle difference from } \\
& \text { bus } 1 \text { to bus } 2
\end{aligned}
$$

For $B_{12}=-B_{22}=10.0$, the locus of points in the $\alpha-V$ space satisfying these constraints for a range of $P$ and $Q$ values are shown in Figure 1. This diagram may be considered as a variation on the classic voltage versus power factor curves described in [1]. A radial line with a fixed sending voltage typically has two solutions for receiving end voltage. This is reflected in Figure 1 by the fact that the $\mathrm{P}$ and $\mathrm{Q}$ constraints have typically two intersections, each corresponding to a powerflow solution. We will refer to these as the "high voltage solution" and "low voltage solution," distinguishing the two by their relative values of voltage magnitude. As shown in Figure 1, for certain critical values of $P$ and $Q$, the two constraint curves become tangent, and only one solution exists. If either $\mathrm{P}$ or $\mathrm{Q}$ is increased further, the powerflow has no solution. At this point, the Jacobian of the two power balance equations must be singular. This observation has been used to justify several existing methods of analyzing voltage collapse. In [4], and later in [5], rigorous bifurcation analyses are carried out for the powerflow equations, indicating that this bifurcation phenomena observed in this simple example will occur in powerflow equations for a multibus network. Many other authors have proposed singularity of the Jacobian of the powerflow equations at the high voltage solution (or a closely related measure) as a test for the onset of voltage collapse [1], [2], [3], [6]. In particular, [6] recommends the use of the smallest singular value of the Jacobian of the powerflow equations as a quantitative measure of proximity to voltage collapse. 
To describe the conditions associated with voltage collapse, it has been suggested that a strictly static framework is not sufficient. In [2] the "semi-dynamic" nature of voltage stability problems is discussed. More recently, [7] and [8] have suggested that particular load dynamics or voltage control dynamics need to be considered. In light of these observations, the question then becomes how to use information about pertinent dynamics of the system to measure proximity to the critical point.

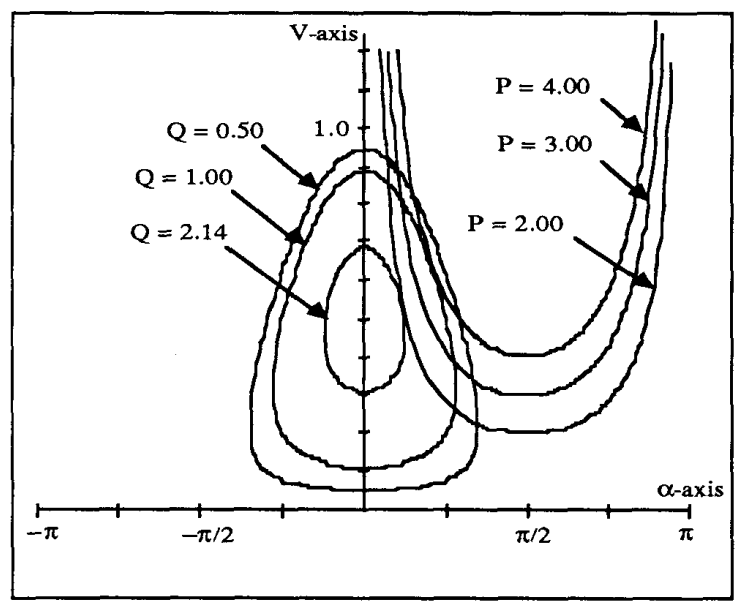

FIGURE 1: Power Balance Constraints in Angle/Voltage Plane

\section{DYNAMIC FRAMEWORK AND ENERGY FUNCTION FOR ONE LINE SYSTEM}

Most reports on incidence of voltage collapse seem to indicate that the collapse was not immediately driven by a large disturbance in the system [9]; lack of an initiating fault is one of the features that distinguishes voltage collapse from transient stability. Instead, the system operating point is moving (on a time scale of minutes to hours), usually under the influence of gradually increasing average load. A key component in analyzing this scenario is the nature of the time variation in the load itself. While the average value of the load may be changing very slowly, one expects that small (a few percent) random variations about this average occur with a broad band frequency spectrum. Research into aggregate load models has repeatedly suggested that such small random effects may be modeled by a white or colored noise term in the load [10], [11]. These random variations should have a negligible effect on a normal, robust operating point, but they may be significant when the operating point is near a critical point. Reference [1] shows that in the operating region near collapse bus voltage is extremely sensitive to changes in load. Part of the premise of this paper is that the interaction of these broad band load variations and the pertinent system dynamics is the driving force that pushes the system "over the edge" to voltage collapse.

To illustrate the interaction of system dynamics with load, we return to the simple one line system. The reader should note that the dynamics in this example do not represent the most general models that can be accommodated by the method. The goal here is to illustrate the basic methodology; the range of allowable models will be discussed later. Suppose bus 1 is a generator bus. We will assume that the real power demand is a constant plus a linear term dependent on bus frequency. This follows the structure preserving model introduced for transient stability analysis in [12]. Using a classical model for the generator, the system equations are then given by:

$$
\begin{aligned}
& M_{g} \dot{\omega}+D_{g} \omega+B_{12} V \sin \left(\delta_{1}-\delta_{2}\right)-P_{M}=0 \\
& -\left(P_{L}+D_{l} \dot{\delta_{2}}\right)=B_{21} V \sin \left(\delta_{2}-\delta_{1}\right) \\
& -Q_{L}=-B_{22} V^{2}-B_{21} V \cos \left(\delta_{2}-\delta_{1}\right)
\end{aligned}
$$

Under the assumptions that $P_{M}=P_{L}$ (generator mechanical power matches active load demand) and $B_{12}=B_{21}$, and recalling the definition of $\alpha$, these may be re-written as:

$$
\begin{aligned}
& \dot{\omega}=-M_{g}^{1} D_{g} \omega-M_{g}^{-1} f(\alpha, V) \\
& \dot{\alpha}=-D_{l}^{-1} f(\alpha, V)+\omega \\
& 0=g(\alpha, V)
\end{aligned}
$$

where

$$
\begin{aligned}
& f(\alpha, V):=B_{12} V \sin (\alpha)-P_{L} \\
& g(\alpha, V):=V^{-1}\left\{Q_{L}-B_{12} V \cos (\alpha)-B_{22} V^{2}\right\}
\end{aligned}
$$

The reader should note that the multiplication by $\mathrm{V}^{-1}$ in (2c) does not affect the desired solutions because voltage magnitudes are always restricted to be strictly positive. The equilibria of (2) are the $(\alpha, V)$ intersection points pictured in Figure 1 , in the $\omega=0$ plane.

The mixed system of differential equations with algebraic constraints given by (2) is a reasonable description of the simple system model in the vicinity of the high voltage operating point, but may not possess a valid solution when voltage magnitudes are very low [13]. For analyzing voltage collapse, one requires a model that is at least qualitatively correct in its behavior over a very wide range of voltage magnitudes. Unfortunately, the state of the art in load modeling is such that we can not expect to have a precise description of load behavior over the entire range of voltages likely to be encountered in voltage collapse. As a first step towards dealing with this problem, we propose the following. We will relax the algebraic constraint of $(2 c)$ in a manner that will lead to behavior nearly identical to the mixed differential/algebraic model near the high voltage equilibrium, but will allow well defined trajectories under very low voltage conditions.

A reactive load model for the power system fulfilling the above requirements was proposed in [13] using singular perturbation techniques adopted from [14]. The approach can be very easily illustrated in the context of the two bus example here. Mathematically, the algebraic equation is singularly perturbed to form a differential equation whose equilibrium is the solution of the original reactive power balance equation. For $(2 \mathrm{c})$, this becomes

$$
\dot{\varepsilon}=-g(\alpha, V)
$$

where $\varepsilon$ is a small positive parameter that controls the speed with which trajectories of voltage magnitude move towards values satisfying reactive power balance. We will show later that the model's ability to predict voltage collapse is independent of the choice of this parameter. From an engineering standpoint, (3) may be interpreted as follows. The load demand is taken as the 
"independent input," and the voltages respond to this input to maintain reactive power balance. The right hand side of (3) is the difference between reactive power delivered by the network and reactive power absorbed by the load. When the load instantaneously demands more reactive power than is being delivered, (3) predicts that the bus voltage drops until power balance is re-established. When excess reactive power is delivered by the network, bus voltage magnitude will increase. The rate of this change varies with $\varepsilon$; for $\varepsilon$ sufficiently small, it is essentially instantaneous and behavior is nearly identical to the original static model. This is qualitatively reasonable behavior for many types of loads 1 . Use of (3) is not advocated in simulating system trajectories; clearly, for $\varepsilon$ small, one would create an unnecessarily stiff set of differential equations to be solved. The point of introducing (3) is to obtain a single model that is physically reasonable over a wide operating range of voltage, thereby facilitating the energy function analysis.

Relaxing the algebraic constraint results in a model ((2a), (2b) and (3)) composed only of ordinary differential equations, and is therefore suited to the application of standard Lyapunov theory. For an equilibrium point of $\left(0, \alpha^{0}, V^{\circ}\right)$, consider the candidate energy function:

$$
\begin{aligned}
& \vartheta(\omega, \alpha, V):=\frac{1}{2} M_{g} \omega^{2} \\
& \text { - } B_{12} V \cos (\alpha)+B_{12} V^{0} \cos \left(\alpha^{0}\right) \\
& -\frac{1}{2} B_{22} V^{2}+\frac{1}{2} B_{22}\left(V^{0}\right)^{2} \\
& \text { - } P_{L}\left(\alpha-\alpha^{0}\right)+Q_{L} \ln \left(\frac{V}{v^{0}}\right) \\
& (\omega, \alpha, V) \\
& =\int\left[M_{g} x, f(y, z), g(y, z)\right] \cdot[d x, d y, d z]^{T} \\
& \left(0, \alpha^{0}, v^{0}\right)
\end{aligned}
$$

Note immediately that $\vartheta(\omega, \alpha, V)$ as selected is independent of $\varepsilon$. The reasoning behind selecting this function becomes clear from the following observations:

Observation 1

Consider the system of differential equations defined by:

$$
\left[\begin{array}{c}
\dot{\omega} \\
\dot{\alpha} \\
\dot{V}
\end{array}\right]=\left[\begin{array}{ccc}
-M_{g}^{-1} D_{g} M_{g}^{-1} & -M_{g}^{-1} & 0 \\
M_{g}^{-1} & -D_{l}^{-1} & 0 \\
0 & 0 & -\frac{1}{\varepsilon}
\end{array}\right] \nabla \vartheta(\omega, \alpha, V)
$$

Straightforward calculations reveal that these are identical to the singularly perturbed model; i.e. (5) is an alternate expression for the system dynamics system defined in (2a), (2b), and (3).

\section{Observation 2}

The rate of change of energy along trajectories of the system is always less than or equal zero. This follows from:

${ }^{1}$ Note that control equipment at the load bus may cause this characteristic to reverse with changes in operating point. The ac side of a HVDC inverter may under some operating conditions display behavior where an increase in reactive power delivered by the network actually lowers bus voltage. Clearly, a more complex model than that proposed above is necessary to handle such situations.

$$
\frac{\mathrm{d} \vartheta}{\mathrm{dt}}=\frac{1}{2} \nabla \vartheta^{\mathrm{T}}(\omega, \alpha, \mathrm{V})\left(\mathbf{A}+\mathbf{A}^{\mathrm{T}}\right) \nabla \vartheta(\omega, \alpha, \mathrm{V})
$$

with $\mathbf{A}$ defined as the $3 \times 3$ matrix appearing on the right hand side of $(5) ;\left(A+\mathbf{A}^{\mathrm{T}}\right)$ is a diagonal negative semi-definite matrix.

\section{Observation 3}

The equilibria of (5) occur only at those points where $\nabla \vartheta=0$. This follows from (5) and the fact that A is nonsingular. This confirms the previous observation that equilibria can occur only where $\omega$ is zero.

\section{Observation 4}

If the linearization of (5) about an equilibrium point is strictly stable (i.e., all eigenvalues have strictly negative real parts), then the Hessian (second derivative) of $\vartheta(\omega, \alpha, V)$ evaluated at the equilibrium has all positive real eigenvalues. This in tum implies that $\vartheta(\omega, \alpha, \vee)$ is locally positive definite about that equilibrium point.

From observations (1)-(4) above, one can conclude that for an operating point that is small disturbance stable, the energy function proposed is formally a Lyapunov function (see [15], theorem (106)). The Lyapunov function defined in (4) and the corresponding dynamics in (5) generalize immediately to systems of arbitrary dimensions by defining $f$ and $g$ to be vector functions whose components are active and reactive power balance equations at each bus. This generalization is discussed in [16] and [17]. However, if one strictly requires that (4) define a true Lyapunov function, this construction does place restrictions on allowable load models. Reactive power demand is allowed to be an arbitrary polynomial in bus voltage magnitude; path independence of the integral in (4) requires that active power demand not depend on voltage magnitudes. However, extensive experience in energy functions for transient stability studies [18] has shown that a path dependent integration may be used in terms such as (4) with the resulting "energy function" still approximating many of the properties of a Lyapunov function. We will adopt that approach here for models having voltage dependent active demands, and hence use the terminology "energy function" rather than Lyapunov function through the remainder of this paper.

Also important to voltage stability studies is the ability to represent on-load tap changing transformer behavior. With active and reactive load given as functions of voltage magnitudes, a static model for tap changing transformer behavior can be incorporated. More detailed modeling that includes time delays in tap switching requires dynamic equations for tap motion such as presented in [19]. The question of whether these can be added to this energy function analysis has not yet been addressed.

To understand the use of the energy function to study voltage collapse, it is useful to review its use in transient stability. In that context, the simplest Lyapunov based stability criterion uses the concept of the closest unstable equilibrium. Roughly, the criteria may be stated as follows. Starting from the post fault equilibrium of interest, expand constant energy contours of the $\vartheta$ function until they intersect another equilibrium point. Evaluate the $\vartheta$ function at this "closest unstable equilibrium point." If the initial energy following the fault is less than this amount, the system will asymptotically return to the desired operating point. This method is generally judged too conservative, for the following reason. The closest unstable equilibrium point represents the lowest saddle 
point by which trajectories may escape the potential energy wel surrounding the stable equilibrium point. A fault that happens to push the system trajectory through this lowest saddle point is a rare, worst case scenario.

However, consider again the conditions associated with voltage collapse. Load is gradually increasing in a way that causes the high voltage equilibrium (normal powerflow solution) to approach the low voltage equilibrium. The increase in load shrinks the potential well and lowers the saddle point that represents the easiest path of escape. The energy function then provides a measure of this ease with which load variations push the system state through this closest unstable equilibrium. The reader familiar with direct methods of transient stability will note that calculating the closest unstable equilibrium point in models that include only active power flow equations has proven computationally prohibitive in large scale systems. This does not prove true in the models used here, due in part to the restrictions that come from adding reactive power balance equations to the model. This issue will be discussed in more detail in Section V.

\section{USE OF THE ENERGY FUNCTION TO ESTIMATE EXIT TIME}

The preceding discussion motivates the following approach to measuring proximity of an operating point to voltage collapse: measure distance by the difference in energy between the high voltage and low voltage solutions of the powerflow. This is fundamentally a nonlinear, "non-local" measure. The questions to be answered in justifying this viewpoint on voltage collapse are the following. First, when driven out of the potential well, do the trajectories in our simple model show rapid collapse of voltage magnitude? Second, do random load variations tend to drive the system out of the potential well when there is no large disturbance? The first question proves easy to answer. If the state exits the potential well in the vicinity of the low voltage equilibrium (which is the easiest path of escape), it will fall in a region where the right hand side of (3) is negative, and the voltage magnitude is driven rapidly down. To answer the second question, we return to the proposition that the time behavior of load should include a small magnitude broad spectrum random term. Conceptually, this effect can be modeled by including a small magnitude white noise (or filtered white noise) term in each $\mathrm{P}$ and $\mathrm{Q}$ load. In this framework, the state of the system becomes a random process, and one can formally define the expected time required for the state to exit the potential well.

The calculation of expected exit times for a randomly perturbed power system models was examined using the theory of large deviations in [17]. The pertinent result may be summarized as follows: for an $\varepsilon$ magnitude white noise disturbance in the loads, the expected time required to leave the potential energy well is proportional to:

$$
\exp \frac{\vartheta\left(\omega^{\mathrm{u}}, \alpha^{\mathrm{u}}, V^{\mathrm{u}}\right)}{\varepsilon^{2}}
$$

where $\left(\omega^{\mathrm{u}}, \alpha^{\mathrm{u}}, \mathrm{V}^{\mathrm{u}}\right)$ represents the closest unstable equilibrium point in the sense defined above. The exit time calculation gives a rigorous means by which different operating points may be ranked in terms of their vulnerability to voltage collapse. However, in the following section, we will examine another, more heuristic application of the energy measure that combines a single energy calculation at the current operating point with a family of energy curves obtained by increasing load from a normal operating region until the critical point of loss of the powerflow solution.

\section{USE OF THE ENERGY FUNCTION TO ESTIMATE PROXIMITY TO VOLTAGE COLLAPSE}

This section develops a security measure that describes distance to voltage collapse in terms of the MW load increase required (under a given assumption on load participation factors) to drive the system to collapse. The first test system consists of two strongly coupled generator buses, numbered 1 and 2, with a weakly coupled load bus (number 3) attached to the second generator. Line 1 links buses 1 and 2, while line 2 links buses 2 and 3. This system was chosen because it is a simple equivalent representation to the type of system which is often prone to voltage collapse.

Consider the following system parameter values:

$$
\mathrm{R} 1=0.005 \mathrm{X} 1=0.05 \mathrm{R} 2=0.02 \mathrm{X} 2=0.06
$$

Assume that all loads are constant $P / Q$. Initially, the generator at bus 2 is off-line and that there is no load at bus 2 . In this case, the energy function can be calculated for any $P / Q$ load at bus three. Figure 2 plots the difference in energy between the high and low voltage powerflow solutions as the load at bus 3 is increased. Beginning at $50 \mathrm{MW}$, the load is increased with a constant $P / Q$ ratio of 2 until the critical collapse point occurs at approximately $\mathrm{P}=242 \mathrm{MW}, \mathrm{Q}=121 \mathrm{MVAR}$

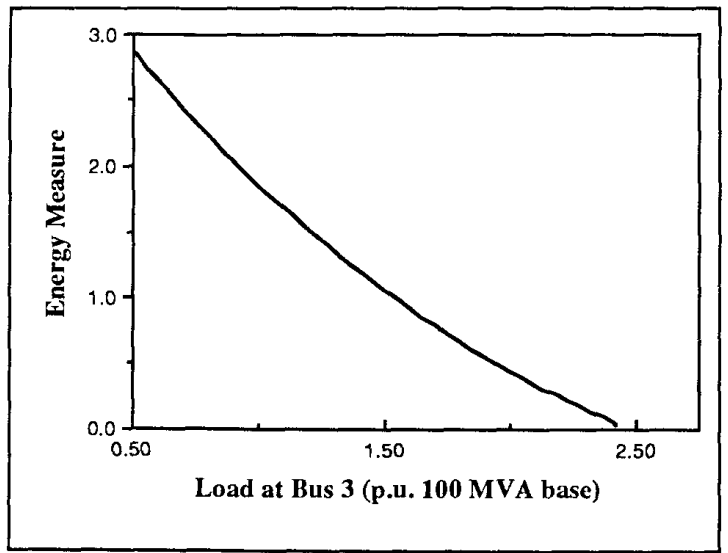

FIGURE 2: Energy Measure versus Load Level

One possible application of the energy values shown in Figure 2 is calculation of the 'expected exit time' discussed in the previous section. However, Figure 2 also suggests a more intuitive measure of system security. By shifting the $x$ axis to align the critical collapse point with zero $\mathrm{MW}$, each energy value can be related to a "distance" in MW to voltage collapse. For an energy value of 0.5 , the distance to collapse is approximately 50 $\mathrm{MW}$; i.e., when the energy value is 0.50 , voltage collapse will occur when the load at bus 3 is increased by $50 \mathrm{MW}$ and 25 MVAR. This suggests the following heuristic test for determining the distance to voltage collapse under general load conditions:

1) Calculate the energy value for a given load at bus 3 . 
2) Use a table containing the Figure 2 data (pre-calculated offline) to determine the 'distance' to voltage collapse. Exploiting the near linearity of Figure 2, relatively few points (powerflow solutions) would be needed to produce a good approximation of this curve.

For this approach to be useful, the shape of the curve in Figure 2 must be relatively insensitive to changes in the operating point of the system. Ideally, one would like to confirm that the near linearity of the curve observed above extends to a variety of systems. Then the approximate distance to voltage collapse for a wide variety of operating points can be computed on-line, using only information from the off-line calculation of a single energy curve. Note that the "energy curve" is simply the difference in energy between the high and low voltage powerflow solutions over a one parameter family of operating points (MW load at bus 3 being the free parameter used in Figure 2). The following examples show the feasibility of such an approach.

First, for the 3 bus system, the operating point is changed by varying the load at bus 2 (with the generator again assumed to be off-line). Figure 3 shows how the energy function varies with the load at bus 3 for different loads at bus 2, with the topmost curve corresponding to a load at bus $2=0$, and is thus simply a repeat of Figure 2. As would be expected, the energy curve is lower as load at bus 2 is increased, since the system is more heavily loaded and therefore less secure. Note that the five curves in Figure 3 are nearly identical up to an $\mathrm{x}$-axis shift, and all vary in a nearly linear fashion with load changes.

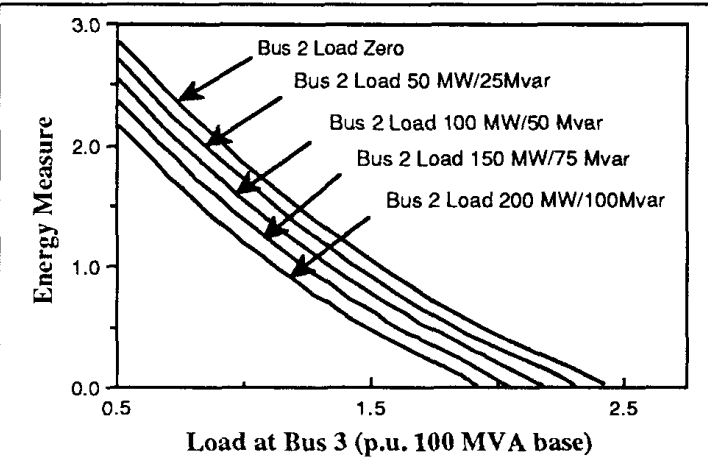

FIGURE 3: Effect of Neighboring Loads on Energy Measure

The next example in the three bus system demonstrates that var limits on generators can also be incorporated using this methodology. In the powerflow calculations employed here, the var output of the generator is typically allowed to vary in order to hold its bus voltage constant; i.e. generator buses are treated as PV. However, if the var limit is reached, the exciter is considered saturated, and the generator's var output is held constant; the bus model changes to $P Q$. This is a standard approach to treating var limits in powerflow calculations. Consider the system operating with load at bus 2 set to zero, while the generator is turned on to provide voltage support (no active power output). One would expect that the more reactive power the generator can provide, the greater the load that can be tolerated at bus 3 before voltage collapse occurs. Figure 4 shows that this is indeed the case. The lowest curve shows zero var output from generator 2 , and is therefore a repeat of Figure 2. The next four curves show how the energy functions varies as the maximum var output of generator 2 is increased in increments of 50 MVAR. During the sequence of powerflow/energy calculations, voltage at bus 2 was held at 1.0 per unit as load ramped up until generator 2 reached its var limit. Thereafter, the var output was held at its maximum. Surprising, the shape of the energy function curve proves insensitive to varying var limits on the generator; the five curves in Figure 4 are again nearly identical up to a shift along the horizontal axis.

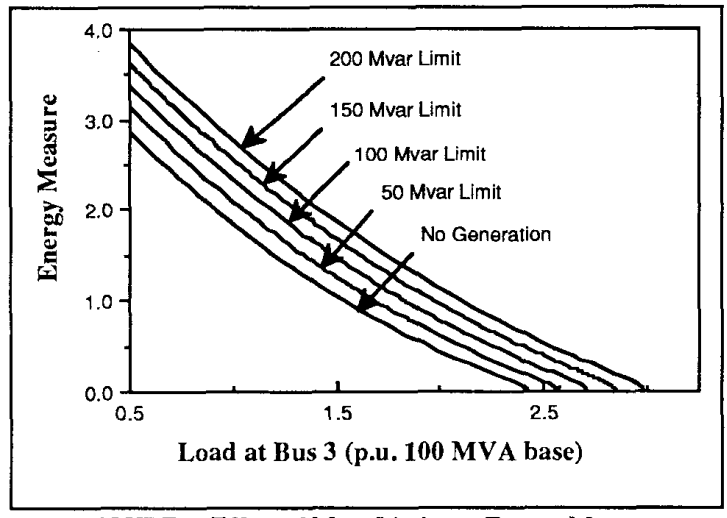

FIGURE 4: Effect of Mvar Limits on Energy Measure

The reader should note that in this example limits on available var support are taken into account, even when the current system operating point does not push generators to these limits. Intuitively, this is because the low voltage solution tends to push all neighboring var sources to their limits, and the var limits reduce the height of the potential energy boundary that the system must cross to experience collapse.

The following examples illustrate the usefulness of the method on a larger standard test system. The New England 30 Bus system used in [20], [21] was chosen as the test system. The voltage collapse scenario examined consisted of increasing the reactive load at bus 11 , while keeping all other loads and generator MW outputs fixed. This is scenario number 1 from [21]. The curve of Figure 5 plots the energy difference as reactive load at bus 11 is increased until voltage collapse occurs. As was the case with the Figure 2, the shape of the curve is approximately linear. This allows for quite reasonable approximations of distance to voltage collapse, even when the current system operating point is quite far from the point of collapse. Figure 5 may be compared to the plots of proximity measures for the same scenario presented in [21].

To complete this example, a series of contingencies from [20] were applied to the system:

1. basecase (same as [21]),

2. after generator 6 is removed,

3. after generator 10 is removed,

4. after generation at buses 2,25 , and 29 is increased by $200 \mathrm{MW}$,

5. after line $(9,30)$ suffers an outage, and

6. after a capacitor bank of 500 MVAR is placed at bus 11 . 
Note that this contingency list is cumulative; for example, number 6 includes all the system changes in numbers 1 through 5 .

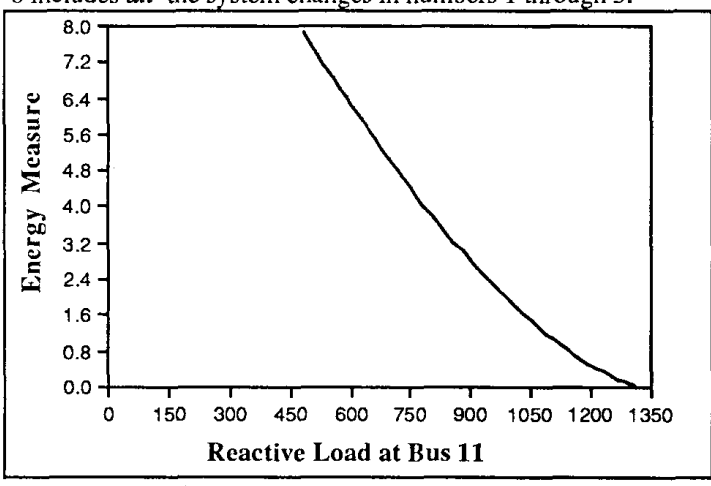

FIGURE 5: Energy Curve 30 Bus System

The lower five curves in Figure 6 display the energy function associated with each contingency as the reactive load at bus 11 is increased. Again, as in the three bus examples, the energy curves remain relatively parallel, though progressively less so as more and more major changes were made to the system structure. This suggests that when rapid on-line evaluation is required, a single energy curve identifying "MW distance to collapse" might be applied to a number of contingencies simply by identifying energy level for a given case.

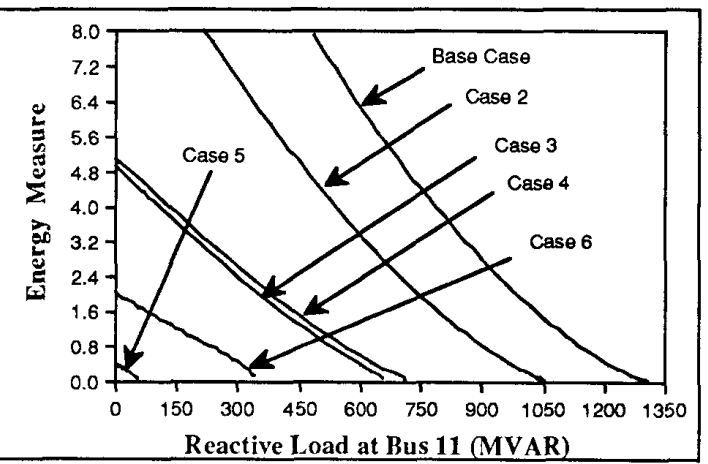

FIGURE 6: Energy Curves 30 Bus System w/contingencies

In an actual system there may be more than one area vulnerable to voltage collapse. Thus a particular low voltage solution and an energy difference to the high voltage solution can be associated with each area of vulnerability. As noted above, experience in locating multiple unstable equilibria in transient stability models might lead the reader to believe that this process will be difficult. However, use of the full powerflow equations with reactive power constraints makes the task much easier in our analysis. Extensive work by Tamura and his co-workers [21]-[24] has confirmed the practicality of locating relevant low voltage solutions in realistically sized networks (43 and 81 buses in [24]). The method used in this example is similar to that described in [22] and [23], where an algorithm is presented to calculate all of a system's low voltage solutions using a rectangular Newton-Raphson technique with the optimal multiplier. Additionally, a "simplified method" is presented to calculate the up to $\mathrm{N}-1$ (where $\mathrm{N}$ is the number of buses in the system) low voltage solutions corresponding to a low initial voltage guess at only a single bus.

References [21] and [24] show that the number of multiple solutions tends to decrease as the system approaches voltage collapse, and that at the point immediately before voltage collapse only a pair of closely located solutions exist. These two are the operable high voltage solution and its low voltage counterpart; the two coalesce at the point of voltage collapse. Calculations in [22] indicate that the "counterpart" solution is typically one of the solutions obtained by the "simplified method." Which solution from the subset of simplified method solutions will become the operating point "counterpart" depends upon the assumed path of load changes that lead to the point of voltage collapse. In an operating or planning environment where many possible patterns of load increase must be considered, energy measures could be calculated for each vulnerable area. The energy measures could then be used to determine the most vulnerable bus groups at the present operating point and also to rank contingencies based upon proximity to voltage collapse in each area. Computationally the calculation of the simplified method solutions at the present operating state would require at most $\mathrm{N}-1$ powerflow solutions. However, the application of localized voltage screening methods ([20] and [25]) to identify vulnerable areas could greatly reduce this figure. The method also has the attractive property that as the system approaches the point of voltage collapse, the number of simplified solutions reduces ultimately to one; i.e., the computational burden of the method actually goes down as the system becomes more heavily loaded.

\section{CONCLUSIONS}

This paper has introduced a new method of assessing vulnerability of a power system operating point to voltage collapse based upon an energy function method. The method offers a smoothly varying measure of proximity to the critical point of voltage collapse which operators might use to anticipate when corrective action is needed before collapse occurs. Var limits on generators are taken into account even before those limits have been reached at the current system operating point.

Computationally, the on-line requirements consist of only one powerflow solution (in addition to the solution of the current operating point) and an associated evaluation of a scalar energy function for each vulnerable bus. Fast screening methods and other heuristic methods could be used to determine the subset of vulnerable buses. If one wishes only to rank various operating points with respect to vulnerability to voltage collapse, one energy evaluation at each operating point (with two power flows each) is needed. To examine a single possible patterns of expected load increase and produce a curve of the type pictured in Figure 5, the off-line calculations require a moderate number of powerflow solutions (roughly ten) to evaluate energy along the "projected path" of operating points. The number of powerflow solutions required to accurately produce a graph such as Figure 5 for a single load pattern depends primarily on the projected path of load increase, not on the size of the network. On the other hand, the number of vulnerable areas of interest (and hence the number of possible "paths") in the network can be expected to grow with network size. If one uses only low voltage powerflow solutions to identify vulnerable groups of buses, the number of power flow calculations grows linearly with the number of buses. However, 
it is likely that heuristic judgements based on engineering experience could significantly reduce the number of bus groups that are judged vulnerable to voltage collapse, thereby reducing the required calculation. Once these energy curves are produced offline, a single energy value calculated on-line can then be used to identify where the system lies on a particular curve.

The method in its existing state of development appears quite promising. Further research would be valuable in several areas. First, more detailed dynamics should be included in the model and associated energy function. In particular, the effects of time delays in on-load tap changing transformers, and the influence HVDC links would be very relevant. Further work on efficient low voltage solutions methods is also important.

\section{ACKNOWLEDGEMENTS}

The support of this work by the National Science Foundation under grants ECS-8611728 and ECS-8857019 is gratefully acknowledged.

\section{REFERENCES}

[1] B.M. Weedy and B.R. Cox, "Voltage stability of radial power links," Proc. IEE, vol. 115, no. 4, pp. 528-536, April 1968.

[2] Y. Tamura, H. Mori and S. Iwamoto, "Relationship between voltage instability and multiple load flow solutions in electric power systems" IEEE Trans. Power App. and Sys., vol. PAS-102, no. 5, pp.1115-1125, May 1983.

[3] P. Kessel and H. Glavitsch, "Estimating voltage stability of a power system," P.I.CA. Proc., pp. 424-430, May 1985.

[4] A. Araposthatis, S. Sastry, and P. Varaiya, "Analysis of the power-flow equation," Int. J. of Electrical Power Energy Systems, vol. 3, pp. 115-126, July 1981.

[5] II.G. Kwatny, A.K. Pasrija, and L.Y. Bahar, "Loss of steady state stability and voltage collapse in electric power systems," Proc. of 24th IEEE C.D.C., pp. 804-811, Dec. 1985.

[6] A. Tiranuchit, et al., "Towards a Computationally Feasible On-Line Voltage Instability Index," PICA Proc., pp. 136-142, Montréal, Québec, May 1987.

[7] R. J. Thomas and A. Tiranuchit, "Dynamic Voltage Instability," Proc. 26th IEEE CDC, pp. 53-58, Los Angeles, CA, Dec. 1987.

[8] M. Ilic and F. Mak, "Mid-Range Voltage Dynamics Modeling with the Load Controls Present," Proc. 26th IEEE $C D C$, pp. 45-52, Los Angeles, CA, Dec. 1987.

[9] C. Barbier and J-P. Barret, "An analysis of phenomena of voltage collapse on a transmission system," Rev. Gen. Elect., vol. 89, no.10, pp. 672-690, Oct. 1980.

[10] F. D. Galiana, E. Handschin and A.R. Fiechter, "Identification of stochastic electric load models from physical data," IEEE Trans. Automatic Control, vol. AC-19, pp. 887-893, Dec. 1974.

[11] C.W. Brice et. al., "Physically based stochastic models of power system loads," U.S. Dept. of Energy Report DOE/ET/29129, Sept. 1982.

[12] A.R. Bergen and D.J. Hill, "A structure preserving model for power systems stability analysis," IEEE Trans. Power App. and Sys., vol. PAS-101, pp. 25-35, Jan. 1981.

[13] C.L. DeMarco and A.R. Bergen, "Application of singular perturbation techniques to power system transient stability analysis," I.S.C.A.S. Proc.,pp. 597-601, Montreal, May 1984 (abridged version); also Electronics Research Laboratory, Memo. No. UCB/ERL M84 7 , U. of CA, Berkeley (complete version).

[14] S.S. Sastry, C.A. Desoer, and P. Varaiya, "Jump Behavior of Circuits and Systems," IEEE Trans. Circuits and Systems, vol. CAS-28, no. 12, Dec. 1982.

[15] M. Vidyasagar, Nonlinear Systems Analysis, Prentice-Hall, Englewood Cliffs, NJ, 1978.

[16] C. L. DeMarco, "A New Method of Constructing Lyapunov Functions for Power Systems," Proc. IEEE Int. Symp. on Circuits and Systems, pp. 905-908, Espoo, Finland, June 1988.

[17] C. L. DeMarco and A. R. Bergen, "A Security Measure or Random Load Disturbances in Nonlinear Power System Models," IEEE Trans. Circuits and Systems, pp. 1546-1557, vol. CAS-34, no. 12 , Dec. 1987.

[18] A. A. Fouad et al., "Direct Transient Stability Analysis Using Energy Functions: Applications to Large Power Networks," IEEE Trans. on Power Systems, vol. PWRS-2, pp. 37-44, Feb. 1987.

[19] C-C. Liu, "Analysis of a Voltage Collapse Mechanism Due to the Effect of On-Load Tapchangers," Proc. IEEE Int. Symp. on Circuits and Systems; pp. 1028-1030, San Jose, CA, May 1986.

[20] R. A. Schlueter, A. G. Costi, J. E. Sekerke and H. L. Forgey, "Voltage Stability and Security Assessment", EPRI Report, El-5967, Project 1999-8, August 1988.

[21] Y. Tamura, K. Sakamoto, Y. Tayama, "Voltage Instability Proximity Index (VIPI) Based on Multiple Load Flow Solutions in Ill-Conditioned Power Systems", Proc. 27th IEEE Conf. Decision and Control, Austin, TX, Dec. 1988.

[22] Y. Tamura, K. Iba and S. Iwamoto, "A Method for Finding Multiple Load Flow Solutions for General Power Systems", IEEE PES Winter Meeting, A 80 043-0, New York, Feb. 1980.

[23] S. Iwamoto and Y. Tamura, "A Load Flow Calculation Method for Ill-Conditioned Power Systems", IEEE Trans. Power App. and Sys., vol PAS-100, pp. 1736-1743, April 1981.

[24] Y. Tamura et. al., "Monitoring and Control Strategies of Voltage Stability Based on Voltage Instability Index", Engineering Foundation Conference on Bulk Power System Voltage Phenomena: Voltage Stability and Security, Potosi, MO, Sep. 1988.

[25] G. C. Ejebe, H. P. Van Meeteren, and B. F. Wollenberg, "Fast Contingency Screening and Evaluation for Voltage Security Analysis," IEEE PES Winter Meeting, WM 161-2, New York, Feb. 1988

Christopher L. DeMarco (S '80, M '85) was born in Derby, Connecticut in 1958. He received his Bachelor of Science degree in Electrical Engineering from the Massachusetts Institute of Technology in June of 1980, and his Ph.D. degree in Electrical Engineering and Computer Sciences from the University of California, Berkeley in May 1985. In January 1985, he joined the faculty of the Department of Electrical and Computer Engineering at the University of Wisconsin-Madison, where he currently holds the rank of Assistant Professor.

Thomas J. Overbye (S '87) was born in Milwaukee, WI in 1960. He received his Bachelor of Science degree from the University of Wisconsin-Madison in 1983. He was employed with Madison Gas and Electric Company from 1983 through 1986. He is currently pursuing a $\mathrm{PhD}$ in Electrical Engineering at the University of Wisconsin-Madison. 


\section{Discussion}

C. O. Nwankpa and S. M. Shahidehpour (Department of Electrical and Computer Engineering, Illinois Institute of Technology, Chicago, Illinois): The authors should be commended for their interesting paper on the energy based security measure to indicate the voltage collapse. We agree with the authors that "...A key component in analyzing this scenario (i.e. voltage collapse) is the nature of the time variation of the load itself" The authors investigated the effect of load variations on the proposed energy measure in a normal operating region before it reaches a critical point.

Would the authors explain as to how multi-parameter (different loads) changes affect the proposed energy based measure? In case loads are not changing monotonically, the energy curves will not exhibit an approximate linear character as in the single parameter case. Even if the measure can exhibit a smoothly varying character in the multi-parameter case, the plotting of "projected path(s)" for eventual on-line use will encompass the consideration of a large number of system configurations which would reflect the possible system behavior

As pointed out by the authors, one of the applications of energy function is in the estimation of the exit time from the domain of attraction of specific operating point. When near a critical point, perturbations of system loads may play a major role in the voltage collapse explanation. A specific security index may indeed take into account various intensities and bandwidths of fluctuations in system loads, however, the approximate proportionality formula given in the paper is for the case of constant white noise intensities for all loads. In reality, due consideration of a whole spectrum of small fluctuations is in order, whose derivation process is described in $[\mathrm{A}]$. In the case of colored noises, one may refer to $[\mathrm{B}, \mathrm{C}]$ Authors' comments on this subject will be appreciated.

The proposed use of a single energy curve identifying the "MW distance to collapse" should be called into question. This is because, as long as the voltage collapse has not been reached, a multitude of stable operating points may refer to a given energy level. In other words, for on-line evaluation different contingencies may refer to completely different energy curves. thereby the procedure of simply identifying the proximity measure by the energy level for a given case is rather questionable. The authors' input on this feature will be appreciated.

It is a known fact, as the paper has also shown, that the voltage instability is the reflection of power system behavior under heavily loaded conditions. Because of this, losses in the transmission network would drastically increase as investigated in [21]. Will the authors consider the incorporation of these losses in the proximity measure since the losses are often of magnitudes that would affect the actual energy function?

A main feature of any eventual on-line voltage collapse proximity measure is the ability to provide local information regarding voltag sensitivities as well as the system-wide information. The proposed security measure is the "MW distance to collapse" for a specific node in the system. So, is this measure capable of judging the overall system's or subsystem " proximity to voltage collapse, and would it be able to consider man possible load pattern of neighboring nodes in its evaluation? Authors comments on these points will be appreciated.

\section{References}

[A] C. O. Nwankpa and S. M. Shahidehpour, "Inclusion of small randon pertubations in a structure preserving model for power systems reliability studies." The $4^{\text {rh }}$ IASTED International Conference: Power High Tech '89, July 1989, Valencia, Spain

[B] C. O. Nwankpa and S. M. Shahidehpour, "A generalized approach to reliability evaluation of a dynamic system," Submitted to IEEE Trans. Aut. Cont.

[C] C. O. Nwankpa and S. M. Shahidehpour, "Colored noise modeling in the reliability evaluation of electric power systems," Submitted to International Journal of Applied Mathematical Modelling.

C. L. Demarco and T. J. Overbye: The discussers bring up a number of interesting points with regard to the use of the energy based security measure. Each paragraph of the discussion raises a distinct point; we will address these in order.

The discussers ask how multi-parameter load changes affect the energy measure, in contrast to the one parameter increase that was used for the sample plots in the paper. The first point to make in this regard is that the energy margin at the current operating state can always be evaluated to give an absolute measure of security; i.e. the instantaneous energy value can be evaluated without projections of system parameter changes. This instantaneous measure of security would probably be the most useful energy calculation in cases where there is very large uncertainty in the load projections for the time horizon of interest. However, we envision many applications where an accurate load projection is available, and the operator wishes to know if the projected load increase will bring the system to an insecure state. In this case, it is reasonable to treat the vector of average load values at each bus as a known function of time. The vector of average load values then depends only on one parameter: time. The value of energy could simply be plotted versus time, as is done with Tamura's VIPI index in [21]. Note that the example used in the paper chose to plot the energy versus a single bus load increase to allow comparison to a large number voltage security measures in the literature that had already used this identical example. The generalization to allow multiple load increases as functions of a single parameter is straightforward.

The second point raised by the discussers relates to the interpretation of the energy measure as an "expected exit time," which was mentioned briefly in the current paper, having been explored in detail in [17]. The discussers question the rationale behind a white noise model for small magnitude load variations. Our reasoning is as follows. The small magnitude random variations in loads at distribution points are the aggregate of many thousands of switching actions on individual customer devices. A reasonable model for the aggregation of such switching events at a distribution bus would be a filtered jump process: small jumps represent each switching event, filtered by the impedance separating the customer device from the distribution point. In [C1] the authors show that under certain assumptions (essentially requiring the switching events to be small magnitude and high frequency-as expected in the power systems context) the exit statistics in a model with white noise disturbances will closely approximate those predicted in a model with filtered jump disturbances. Moreover, several detailed studies of random disturbance models in power system loads have confirmed that white noise approximations are reasonable in many applications [10]. [11]. However, if one wished to consider colored noise processes, as the discussers indicate they have in [B], [C] (unfortunately these references were not available in the open literature at the time of this writing), the approach seems straightforward. One would augment the power system dynamic model with a fictitious filter at each load bus. A white noise process is input to this filter; the output is a colored noise process that would serve as the load disturbance. The critical question in such an approach is whether the optimal control problem associated with the expected exit time calculation remains solvable in closed form (see [17]).

The discussers next comment that "for on-line evaluation different contingencies may refer to completely different energy curves." This is indeed the case, as we attempted to indicate by the six different energy curves for the six contingencies illustrated in Figure 6. The contingency of interest must be known to determine the resulting operating point, and the operating point must be known to evaluate the energy. The paper does comment that once the energy is known, rapid (and certainly approximate on-line evaluation of a single energy curve might be used for several contingencies. This is meant only as a rough approximation, to exploit the observed phenomenon that the energy curves are nearly linear and close to being parallel for a number of contingencies. The discussers are justified in questioning the accuracy of such an approximation if a contingency drastically alters the network structure.

The discussers also ask if we will consider incorporation of losses in the proposed proximity measure. This is already done in the current formulation, though it was not adequately stressed in the paper. As noted in the paper, the function used is formally a Lyapunov function for the system model only if transfer conductances are neglected. However, the thirty bus test system examined in the paper did include realistic transfer conductance values in all calculations, so we were careful to use the terminology "energy function" when referring to the security measure proposed. Even though the function itself does not depend directly on $G_{i j}$ parameters, the energy difference depends indirectly on the line conductances though the high and low voltage power flow solutions (which change with conductance values). Therefore, we would argue that loss effects are already incorporated in the security measure as described in the paper.

In their final point, the discussers interpret the results of the paper as proposing "MW distance to collapse" for a specific node in the system as the only use of the energy based security measure, and ask if such a measure is capable of judging the overall system's proximity to collapse. It was not our intention that MW distance to collapse for load increasing only at a single node be the main application of the energy measure. As noted above, we chose to focus on this case because it allowed direct comparison to results for this scenario already in the literature. We apologize if overemphasis on this simple application prevented inclusion of more general uses of the energy based security measure. For example, to obtain sensitivity information, we would propose evaluating the first and second 
order terms in a Taylor expansion of the energy margin (note that this would require evaluation of terms both at the operating point and the unstable equilibrium) as is done for transient stability application of energy functions in [C2].

\section{References}

[Cl] H. J. Kushner, "Robustness and Approximation of Escape Times and Large Deviations Estimates for Systems with Small Noise
Effects," SIAM J. Appl. Math., vol. 44, no 1, pp. 160-182, Feb. 1984.

[C2] V. Vittal, E-Z. Zhou, C. Hwang, and A. A. Fouad, "Derivation of Stability Limits Using an Analytical Sensitivity of the Transient Energy Margin," IEEE Power Engineering Society Winter Power Meeting, Paper \#89 WM 207-2.

Manuscript received August 28, 1989. 